\title{
Evidence for successional development in Antarctic hypolithic bacterial communities
}

\author{
Thulani P Makhalanyane ${ }^{1,2}$, Angel Valverde ${ }^{1,2}$, Nils-Kåre Birkeland ${ }^{3}$, Stephen C Cary ${ }^{4}$, \\ I Marla Tuffin ${ }^{2}$ and Don A Cowan ${ }^{1,2}$ \\ ${ }^{1}$ Department of Genetics, Centre for Microbial Ecology and Genomics, University of Pretoria, Pretoria, \\ South Africa; ${ }^{2}$ Institute for Microbial Biotechnology and Metagenomics, University of the Western Cape, \\ Cape Town, South Africa; ${ }^{3}$ Department of Biology, Centre for Geobiology, University of Bergen, Bergen, \\ Norway and ${ }^{4}$ Department of Biological Sciences, University of Waikato, Hamilton, New Zealand
}

The ISME Journal (2014) 8, 952; doi:10.1038/ismej.2013.230

Correction to: The ISME Journal (2013) 7, 2080-2090; doi:10.1038/ismej.2013.94; published online 13 June 2013

Since the publication of this article, the authors have noticed that one of the co-authors' (Prof M Tuffin) is incorrectly affiliated to the following address: Department of Genetics, Centre for Microbial Ecology and Genomics, University of Pretoria, Pretoria, South Africa. The correct affiliation is shown above.

The Authors would like to apologise for any inconvenience this may have caused.

\section{Using network analysis to explore co-occurrence patterns in soil microbial communities}

\author{
Albert Barberán, Scott T Bates, Emilio O Casamayor and Noah Fierer \\ The ISME Journal (2014) 8, 952; doi:10.1038/ismej.2013.236 \\ Correction to: The ISME Journal (2012) 6, 343-351; \\ doi:10.1038/ismej.2011.119; published online 8 \\ September 2011 \\ The missing file appears in the online version of this \\ corrigendum. \\ The authors would like to apologise for any \\ inconvenience this may have caused.
}

Since the publication of this article, the authors have noticed that there is a missing supplementary file. 